\title{
Evaluation of Reception Facilities for Ship-generated Waste
}

\author{
Sylvia Encheva \\ Stord/Haugesund University College \\ Bjørnsonsg. 45, \\ 5528 Haugesund, \\ Norway
}

\begin{abstract}
Waste management plans usually address all types of ship-generated waste and cargo residues originating from ships calling at ports. Well developed waste management plan is a serious step towards reduction of the environmental impact of ship-generated waste. Such important and at the same time complex considerations can be supported by application of modern mathematical theories. Evaluation of waste management plans based on application of grey theory is presented in this work.
\end{abstract}

Keywords-Waste management, grey theory, assessments

\section{INTRODUCTION}

Waste management plans usually address all types of ship-generated waste including sewage, and cargo residues originating from ships. The volume of waste produces pressure on the environment, particularly with respect to ship-generated waste disposal at home ports and ports of call. Well developed waste management plan is a serious step towards reduction of the environmental impact of ship-generated waste. Obviously, there is a serious need for research on developing intelligent tools for evaluating such plans considering their importance and complexity.

Boolean logic is often used in the process of decision making, [4] and [16]. Thus if a response does not appear to be necessarily true, the system selects false. While Boolean logic appears to be sufficient for most everyday reasoning, it is certainly unable to provide meaningful conclusions in presence of inconsistent and/or incomplete input [5], [6]. This problem can be resolved by applying many-valued logic.

In real life situations qualities are often assessed by using linguistic terms. In order to facilitate such a process which is usually based on incomplete information we propose Grey theory, [7]. This theory is particularly useful with respect to working in situations where the information about elements (or parameters) is incomplete, the information about structure is incomplete, the information about boundaries is incomplete, and the behavior information of movement is incomplete. Occurrence of incomplete information is the main reason of being grey.

The rest of the paper is organized as follows. Basic terms and concepts are presented in Section II. The main results are described in Section III. Section IV contains the conclusion of this work.

\section{BACKGROUND}

Grey theory is an effective method used to solve uncertainty problems with discrete data and incomplete information. The theory includes five major parts: grey prediction, grey relational analysis, grey decision, grey programming and grey control, [2], [3], and [8]. A quantitative approach for assessing the qualitative nature of organizational visions is presented in [10].

The Grey theory in this work follows [7].

Definition 1: A grey system is defined as a system containing uncertain information presented by a grey number and grey variables.

Definition 2: Let $X$ be the universal set. Then a grey set $G$ of $X$ is defined by its two mappings $\bar{\mu}_{G}(x)$ and $\underline{\mu}_{G}(x)$.

$$
\left\{\begin{array}{l}
\bar{\mu}_{G}(x): x \rightarrow[0,1] \\
\underline{\mu}_{G}(x): x \rightarrow[0,1]
\end{array}\right.
$$

$\bar{\mu}_{G}(x) \geq \underline{\mu}_{G}(x), x \in X, X=R, \bar{\mu}_{G}(x)$ and $\underline{\mu}_{G}(x)$ are the upper and lower membership functions in $G$ respectively.

When $\bar{\mu}_{G}(x)=\underline{\mu}_{G}(x)$, the grey set $G$ becomes a fuzzy set. It shows that grey theory considers the condition of the fuzziness and can deal flexibly with the fuzziness situation.

The grey number can be defined as a number with uncertain information. For example, the ratings of attributes are described by the linguistic variables; there will be a numerical interval expressing it. This numerical interval will contain uncertain information. A grey number is often written as $\otimes G$, $\left(\otimes G=\left.G\right|_{\underline{\mu}} ^{\bar{\mu}}\right)$.

Definition 3: Lower-limit, upper-limit, and interval grey numbers.

$\otimes G=[\underline{G}, \infty]$ - if only the lower limit of $G$ can be possibly estimated and $G$ is defined as a lower-limit grey number.

$\otimes G=[-\infty, \bar{G}]$ - if only the upper limit of $G$ can be possibly estimated and $G$ is defined as a upper-limit grey number.

$\otimes G=[\underline{G}, \bar{G}]$ - the lower and upper limits of $G$ can be estimated and $G$ is defined as an interval grey number.

Grey number operation is an operation defined on sets of intervals, rather than real numbers. Some basic operation 
laws of grey numbers $\otimes G_{1}=\left[\underline{G}_{1}, \bar{G}_{1}\right]$ and $\otimes G_{2}=\left[\underline{G}_{2}, \bar{G}_{2}\right]$ on intervals where the four basic grey number operations on the interval are the exact range of the corresponding real operation follow:

$$
\begin{aligned}
& \otimes G_{1}+\otimes G_{2}=\left[\underline{G}_{1}+\underline{G}_{2}, \bar{G}_{1}+\bar{G}_{2}\right] \\
& \otimes G_{1}-\otimes G_{2}=\left[\underline{G}_{1}-\bar{G}_{2}, \bar{G}_{1}-\underline{G}_{2}\right] \\
& \left.\otimes G_{1} \times \otimes G_{2}=\bar{G}_{1} \underline{\min }_{\left(G_{1}\right.} \underline{G}_{1} \underline{G}_{2}, \underline{G}_{1} \bar{G}_{2}, \bar{G}_{1} \underline{G}_{2}, \bar{G}_{1} \bar{G}_{2}\right), \\
& \left.\max \left(\underline{G}_{1} \underline{G}_{2}, \underline{G}_{1} \bar{G}_{2}, \bar{G}_{1} \underline{G}_{2}\right)\right]
\end{aligned}
$$

$$
\otimes G_{1} \div \otimes G_{2}=\left[\underline{G}_{1}, \bar{G}_{1}\right] \times\left[\frac{1}{\underline{G}_{2}}, \frac{1}{\bar{G}_{2}}\right]
$$

The length of a grey number $\otimes G$ is defined as

$$
L(\otimes G)=[\bar{G}-\underline{G}] .
$$

Definition 4: [7] For two grey numbers $\otimes G_{1}=\left[\underline{G}_{1}, \bar{G}_{1}\right]$ and $\otimes G_{2}=\left[\underline{G}_{2}, \bar{G}_{2}\right]$, the possibility degree of $\otimes G_{1} \leq \otimes G_{2}$ can be expressed as follows:

$$
P\left\{\otimes G_{1} \leq \otimes G_{2}\right\}=\frac{\max \left(0, L^{\star}-\max \left(0, \bar{G}_{1}-\underline{G}_{2}\right)\right)}{L^{\star}}
$$

where $L^{\star}=L\left(\otimes G_{1}\right)+L\left(\otimes G_{2}\right)$.

For the position relationship between $\otimes G_{1}$ and $\otimes G_{2}$, there exist four possible cases on the real number axis. The relationship between $\otimes G_{1}$ and $\otimes G_{2}$ is determined as follows:

- If $\underline{G}_{1}=\underline{G}_{2}$ and $\bar{G}_{1}=\bar{G}_{2}$, we say that $\otimes G_{1}$ is equal to $\otimes G_{2}$, denoted as $\otimes G_{1}=\otimes G_{2}$. Then $P\left\{\otimes G_{1} \leq\right.$ $\left.\otimes G_{2}\right\}=0.5$.

- If $\underline{G}_{2}>\bar{G}_{1}$, we say that $\otimes G_{2}$ is larger than $\bar{G}_{1}$, denoted as $\otimes G_{2}>\otimes G_{1}$. Then $P\left\{\otimes G_{1} \leq \otimes G_{2}\right\}=1$.

- If $\bar{G}_{2}>\bar{G}_{1}$, we say that $\otimes G_{2}$ is smaller than $\bar{G}_{1}$, denoted as $\otimes G_{2}<\otimes G_{1}$. Then $P\left\{\otimes G_{1} \leq \otimes G_{2}\right\}=0$.

- If there is an inter-crossing part in them, when $P\left\{\otimes G_{1} \leq \otimes G_{2}\right\}>0.5$, we say that $\otimes G_{2}$ is larger than $\bar{G}_{1}$, denoted as $\otimes G_{2}>\otimes G_{1}$. When $P\left\{\otimes G_{1} \leq \otimes G_{2}\right\}<0.5$, we say that $\otimes G_{2}$ is smaller than $\bar{G}_{1}$, denoted as $\otimes G_{2}<\otimes G_{1}$.

Suppose a decision group has $K$ persons, then the attribute weight of attribute $Q_{j}$ can be calculated as

$$
\otimes w_{j}=\frac{1}{K}\left[\otimes w_{j}^{1}+\otimes w_{j}^{2}++\otimes w_{j}^{K}\right]
$$

where $\otimes w_{j}^{K}, j=1,2, \ldots, n$ is the attribute weight of $K$-th decision maker and can be described by grey number $\otimes w_{j}^{K}=\left[\underline{w}_{j}^{K}, \bar{w}_{j}^{K}\right]$.

The rating values are

$$
\otimes G_{i j}=\frac{1}{K}\left[\otimes G_{i j}^{1}+\otimes G_{i j}^{2}++\otimes G_{i j}^{K}\right]
$$

where $\otimes G_{i j}^{K}, i=1,2, \ldots, m, j=1,2, \ldots, n$ is the attribute rating value of $K$-th decision maker and can be described by the grey number $\otimes G_{i j}^{K}=\left[\underline{G}_{i j}^{K}, \bar{G}_{i j}^{K}\right]$.

The weighted normalized grey decision matrix can be established as

$$
D^{\star}=\left[\begin{array}{llll}
\otimes V_{11} & \otimes V_{12} & \cdots & \otimes V_{1 n} \\
\otimes V_{21} & \otimes V_{22} & \cdots & \otimes V_{2 n} \\
& & \cdots & \\
\otimes V_{m 1} & \otimes V_{m 2} & \cdots & \otimes V_{m n}
\end{array}\right]
$$

where $\otimes V_{i j}=\otimes G_{i j} \times \otimes w_{j}$.

$$
S^{\max }=\left\{\begin{array}{l}
{\left[\max _{1 \leq i \leq m} \underline{V}_{i 1}, \max _{1 \leq i \leq m} \bar{V}_{i 1}\right]} \\
{\left[\max _{1 \leq i \leq m} \underline{V}_{i 2}, \max _{1 \leq i \leq m} \bar{V}_{i 2}\right],} \\
\ldots, \\
{\left[\max _{1 \leq i \leq m} \underline{V}_{i n}, \max _{1 \leq i \leq m} \bar{V}_{i n}\right]}
\end{array}\right\}
$$

The grey possibility degree between plan alternatives in set $P l=\left\{P l_{1}, P l_{2}, \ldots, P l_{m}\right\}$ and ideal referential plan alternative $P l^{\max }$.

$$
P\left\{P l_{i} \leq P l^{\max }\right\}=\frac{1}{n} \sum_{1 \leq j \leq n} P\left\{\otimes V_{i j} \leq \otimes G_{j}^{\max }\right\}
$$

A smaller $P\left\{P l_{i} \leq P l^{\max }\right\}$ implies worse ranking order of $P l_{i}$.

A historical review and bibliometric analysis of grey system theory is presented in [14]. Application of grey theory for predication of electric power demand can be seen in [13].

The Analytic Hierarchy Process (AHP), [11] facilitates development of a hierarchical structure of complex evaluation problems. This way subjective judgment errors can be avoided and an increase of the likelihood for obtaining reliable results can be achieved. AHP employs paired comparisons in order to obtain ratio scales. Both actual measurements and subjective opinions can be used in the process. Grey relational analysis method and analytic network process [12] approach were used in [9].

\section{DECISION-MAKING}

The regulation on waste delivered to shore enforced by 2004 requires vessels entering ports within the European Economic Community (EEC) to report current status of waste on-board. This includes the amount of waste being produced, delivered in port, and planned to be delivered in next port of call. Prior to arrival all the required data has to be delivered to port authorities. Such information is of special interest regarding environmental reporting.

Cruise ships often generate waste that prevails their maximum storage capacity long before they have access to shore 
TABLE I. ATtRIBUtE WEIGHTS

\begin{tabular}{ll}
\hline \multicolumn{1}{c}{ Scale } \\
\hline Not satisfactory & {$[0.0,0.2]$} \\
Somewhat atisfactory & {$[0.21,0.4]$} \\
Average & {$[0.41,0.5]$} \\
Good & {$[0.51,0.8]$} \\
Very good & {$[0.81,0.9]$} \\
Exellent & {$[0.91,1.0]$} \\
\hline
\end{tabular}

waste disposal facilities. According to MARPOL 73/78 discharge of treated waste water is allowed $4 \mathrm{Mi}$ off shore, while some regional port policies require discharge treated waste water $12 \mathrm{Mi}$ off shore.

Establishment and operation of reception facilities for shipgenerated waste is a very important regarding protection of the external environment. The reception facilities can be fixed, floating and mobile units. Examples of ship-generated waste include oily waste, sewage, cargo residues and garbage.

While large oil spills in the see are followed by marine pollution authorities, small spills cased by pumping oily bilge water overboard and refueling receive considerably less attention. They however have also negative effects on the marine environment. Marine bilge pump out services are to be used instead of pumping oily bilge water overboard. The latter may contain diesel and petrol, as well as lubricant and hydraulic oils.

Reception facilities are often ranked according to availability of the pre-treatment equipment and processes, methods of recording actual use of the port reception facilities, methods of recording amounts of received and disposed ship-generated waste and cargo residues. The impact of cruise ship generated waste on home ports and ports of call is studied in [1].

Actual use of reception facilities is sometimes stimulated by the so-called "no-special-fee" system. Thus 'fees covering the cost of the reception, handling and final disposal of shipgenerated wastes are included in the harbor fee or otherwise charged to the ship, irrespective of whether any wastes are actually delivered', [17]. Recent port waste management plan for ship-generated waste can be found in [18].

In this work five plan alternatives $P l 1, P l 2, P l 3, P l 4$ and $P l 5$ are ranked with respect to four attributes. These attributes address provision of facilities to receive $A 1$, treat $A 2$, safely dispose of ship-generated waste $A 3$, and use of green energy $A 4$. We however are not to provide all numerical details due to agreed upon anonymity restrictions. Instead we present a graphical illustration for comparing the plan alternatives.

Further on we use the rule: the grater values the better. The calculations are done based on real data and according to the theory presented in [7]. This means calculating of linguistic ratings for weights and linguistic ratings for attributes, first and building a weighted normalized decision table afterwards. Linguistic ratings for applied weights presented in Table I and weighted normalized decision table is shown in Table II.

The possibility degrees are $P\left\{P l_{i} \leq P l^{\max }\right\}=$ $\{0.65,0.78,0.72,0.61,0.67\}$. In other words $P l 2>P l 3>$ $P l 5>P l 1>P l 4$. According to the performed calculations the second alternative should be chosen.
TABLE II. WEIGHTED NORMALIZED DECISION TABLE

\begin{tabular}{ccccc}
\hline & A1 & A2 & A3 & A4 \\
\hline P11 & {$[6.41,6.83]$} & {$[3.46,3.84]$} & {$[6.25,6.81]$} & {$[3.52,3.86]$} \\
P12 & {$[5.46,5.72]$} & {$[5.34,5.75]$} & {$[7.32,7.57]$} & {$[4.14,4.76]$} \\
P13 & {$[4.34,4.66]$} & {$[5.11,0.23]$} & {$[5.75,6.13]$} & {$[6.25,6.78]$} \\
P14 & {$[4.93,5.28]$} & {$[4.33,4.62]$} & {$[4.32,4.74]$} & {$[4.93,5.08]$} \\
P15 & {$[3.75,4.12]$} & {$[4.83,4.91]$} & {$[5.14,5.63]$} & {$[5.55,6.12]$} \\
\hline
\end{tabular}

Aि1

Fig. 1. Plan alternative $P l 2$ and attributes

Applying grey theory in our case results in ordering proposed plan alternatives when all attributes are taken in consideration. In order to make decisions some authorities are interested to see how different plan alternatives are ranked with respect to each attribute. We answer that question in Fig. 1. Since the second alternative $P l 2$ is listed as the best according the executed calculations, we show where $P l 2$ is placed with respect to each attribute. $P l 2$ appears to be the best alternative according to $A 2$ and $A 3$, and is number two according to $A 1$, and number four according to $A 4$. Alternative $P l 3$ is highlighted Fig. 2.

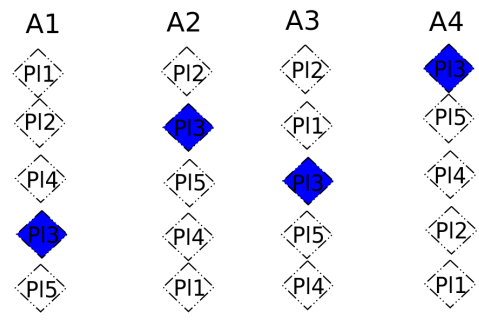

Fig. 2. Plan alternative $P l 3$ and attributes

Similar figures can be made for alternative $P l 5$ if for some reasons alternatives $P l 2$ and $P l 3$ cannot be accepted.

Instead of developing graphical representations one can study the weighted normalized decision table. The latter however proves to be quite difficult when populated with larger amount of data.

\section{CONCLUSION}

Quite often different elements in waste management plans are evaluated independently of each other, which leads to multi-criteria decision inconsistencies. The presented approach can be used to evaluate all elements in such plans and compare those plans in order to make an optimal decision.

We have also compared the five plans applying Analytic Hierarchy Process, [15]. The outcomes conforms what has already been obtained with the Grey theory approach. 


\section{REFERENCES}

[1] N. Butt, The impact of cruise ship generated waste on home ports and ports of call: A study of Southampton, Marine Policy, vol.31(5), pp. 591-598, 2007.

[2] J. L. Deng, Control problems of grey systems, System and control letters, vol. 5, 288-294, 1982.

[3] J. L. Deng, Introduction to grey system theory, Journal of grey systems, 1, vol. 1-24, 1989

[4] R. L. Goodstein, Boolean Algebra, Dover Publications, 2007.

[5] E. Gradel, M. Otto and E. Rosen, Undecidability results on two-variable logics, Archive of Mathematical Logic, vol. 38, pp. 313-354, 1999.

[6] N. Immerman, A. Rabinovich, T. Reps, M. Sagiv and G. Yorsh, The boundery between decidability and undecidability of transitive closure logics, In: CSL'04, 2004.

[7] S. Liu and Y. Lin, Grey information, Springer, 2006

[8] Y.C. Hu, Grey relational analysis and radical basis function network for determining costs in learning sequences, Applied mathematics and computation, vol. 184, pp. 291-299, 2007.

[9] C. Mi and W. Xia, Prioritizing technical requirements in QFD by integrating grey relational analysis method and analytic network process approach, Grey Systems: Theory and Application, vol. 5(1), pp. 117 126, 2015

[10] F. Rahimnia, M. Moghadasian and E. Mashreghi, Application of grey theory organizational approach to evaluation of organizational vision, Grey Systems: Theory and Application, vol. 1, (1), pp. 33-46, 2011.

[11] T. L. Saaty, Principia Mathematica Decernendi: Mathematical Principles of Decision Making, Pittsburgh, Pennsylvania: RWS Publications, 2010.

[12] T. L. Saaty, M. S. Ozdemir and J. S. Shang, The Rationality of Punishment - Measuring the Severity of Crimes: An AHP-Based Ordersof-Magnitude Approach, International Journal of Information Technology and Decision Making, vol. 14(1), pp. 5-16, 2015.

[13] X. Shen and Z. Lu, The Application of Grey Theory Model in the Predication of Jiangsu Province's Electric Power Demand, 2nd AASRI Conference on Power and Energy Systems (PES2013), AASRI Procedia, vol. 7, pp. 81-87, 2014.

[14] M-S. Yin, Fifteen years of grey system theory research: A historical review and bibliometric analysis, Expert Systems with Applications, vol. 40(7), pp. 2767-2775, 2013.

[15] J. Wang, Multi-criteria decision-making approach with incomplete certain information based on ternary AHP, Journal of Systems Engineering and Electronics, vol. 17(1), pp. 109-114, 2006.

[16] J. E. Whitesitt, Boolean Algebra and Its Applications, Dover Publications, 1995.

[17] http://www.helcom.fi/shipping/waste/en_GB/waste/

[18] http://www.bristolport.co.uk/sites/default/pfiles/files/bpc-wastemanagement-plan-2013-issue-3-revision-2-final.pdf 